\title{
Idiopathic chylopericardium
}

\author{
A case report from Saudi Arabia
}

Abeer M. Kawthar, MRCP.

\begin{abstract}

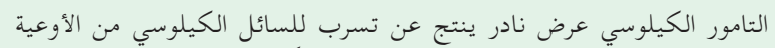

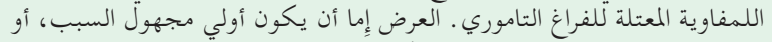

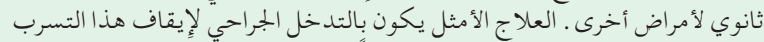

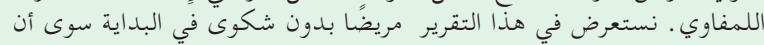

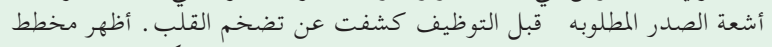

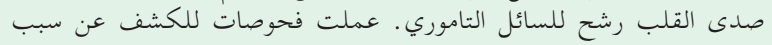

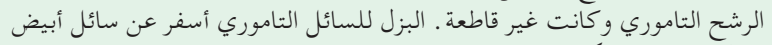

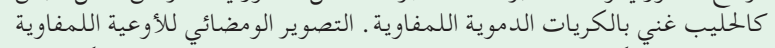

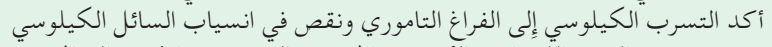

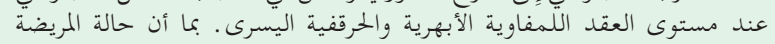

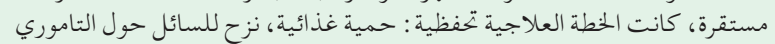

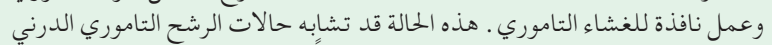

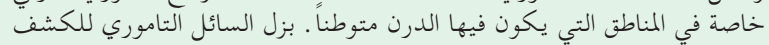
عن الكيلّوس التاموري ذو أهمية بالغة .
\end{abstract}

Chylopericardium is a rare clinical condition in which chyle leaks into the pericardial space owing to lymphatic system derangement. It can be idiopathic or occur secondarily to another clinical condition. Optimal management is accomplished through surgical intervention to impede leaks and drain fluid. In this report, we discuss an initially asymptomatic patient with a pre-employment chest radiograph that revealed cardiomegaly. An echocardiogram revealed pericardial effusion. Medical workup was unable to determine secondary causes for effusion. Pericardiocentesis revealed milky-colored fluid with a high lymphocyte count, and a lymphoscintigraphy lymphangiogram revealed a leak into the pericardium and decreased flow through left iliac and left periaortic lymph nodes. Since the patient was asymptomatic, she was treated conservatively with diet changes, pericardial drainage, and a pericardial window procedure. This type of case risks being misdiagnosed as tuberculosis, especially in countries where tuberculosis is endemic. In such cases, assessing pericardial fluid for chyle is crucial.

Keywords: chylopericardium, pericadiocentesis, pericardial window

Saudi Med J 2020; Vol. 41 (3): 304-308 doi: 10.15537/smj.2020.3.24960
From the Department of Medicine, King Abdulaziz University Hospital, Jeddah, Kingdom of Saudi Arabia.

Received 9th October 2019. Accepted 7th January 2020.

Address correspondence and reprint request to: Dr. Abeer $M$. Kawthar, Assistant Professor, Department of Medicine, King Abdulaziz University Hospital, Jeddah, Kingdom of Saudi Arabia. E-mail:abeer.kawthar@gmail.com

$\mathrm{I}$ diopathic chylopericardium is a rare clinical condition. ${ }^{1}$ Groves and Effler (1954) used the term primary chylopericardium for the first time, defining a case of isolated chyle accumulation in the pericardium without an apparent cause. ${ }^{2}$ Xue Yu et al ${ }^{1}$ conducted a literature review of idiopathic chylopericardium cases reported between 1950 and 2015. A total of 104 cases were identified. There was no gender predilection. The mean age of presentation was 27.95 \pm 16.50 years. Approximately, $39.4 \%$ of the patients were asymptomatic. Shortness of breath was the main complaint in symptomatic patients. Nearly half of the patients who were treated conservatively responded well to the treatment, while the rest required surgical intervention. ${ }^{1}$ Secondary causes of chylopericardium such as lymphangiomas, cystic hygromas, trauma, cardiac or thoracic surgery, malignancy, radiation exposure, or thrombosis of the subclavian vein must be excluded before the diagnosis of idiopathic chylopericardium can be made. ${ }^{2}$ Theoretically, chylopericardium is caused by backflow of lymphatic fluid from the thoracic duct into the pericardial space due to high pressure, abnormal lymphatic vessel wall with valve incompetence, lymphatic obstruction, or lymphangiectasis. ${ }^{1}$ The clinical presentation is related to the severity of chylopericardium; the disorder can be either asymptomatic or accompanied by symptoms including cough, dyspnea, chest pain, or cardiac tamponade. ${ }^{1}$ The diagnosis of chylopericardium can be confirmed by the analysis of pericardial fluid and by lymphangiogram. The chylopericardial fluid is milky 
white and high in triglycerides $(>5.56 \mathrm{mmol} / \mathrm{L})$; it has a triglyceride/cholesterol ratio that is less than one and tests negatively for cultures and cytology. Lymphocytes are the predominant cells present, and fluid tests should be positive for fat lobules using the Sudan III stain. ${ }^{2}$ A lymphangiogram will confirm the leak in the pericardium and may show abnormal communication. ${ }^{1}$ Idiopathic chylopericardium, if left untreated, may cause secondary infections, cardiac tamponade, or constrictive pericarditis. ${ }^{2}$ Management of the disease can be either conservative or surgical. ${ }^{1,3}$ Conservative management strategies includes the administration of a low-fat diet rich in medium-chain triglycerides, total parenteral nutrition, octreotide to decrease fat absorption from the intestine, and a pericardial drain. Dietary fat, mainly, long-chain fatty acids are absorbed by lymphatics and returned back to the circulation through the thoracic duct. A low-fat diet reduces chyle formation. Medium chain fatty acids are absorbed directly into the portal system and do not need lymphatic transport. ${ }^{2}$ Total parenteral nutrition is delivered directly into the blood stream, and it prevents malnutrition from continuous chylopericardium drainage. Octreotide is a somatostatin analogue which reduces intestinal absorption of fat. ${ }^{5}$ The disadvantages of conservative management are their high failure rate $(60 \%)$, risk of malnutrition, risk of metabolic and immunological abnormalities due to the continuous leakage of chyle, and risk of subsequent constrictive pericarditis. Surgical management includes pericardiectomy, which has the risk of re-accumulation of fluid, a combination of thoracic duct ligation above the diaphragm and pericardial window, and videoassisted thoracic surgery. ${ }^{1,4}$ In developing countries, tuberculosis is considered as a common cause of pericardial effusion, ${ }^{6}$ and patients are sometimes treated empirically with anti-tuberculosis antibiotics as a therapeutic trial if no other cause of pericardial effusion can be found. In this report, we discuss a case of primary chylopericardium, in which the assessment of chyle was of the utmost importance for achieving an accurate diagnosis of chylopericardium. The observed effusion may have been confused with tuberculosis in such a patient if such an assessment had not been performed. In addition, the lymphoscintigraphic lymphangiogram confirmed the leak into the pericardial space and suggested a lymphatic flow obstruction of the left iliac

Disclosure. Authors have no conflict of interests, and the work was not supported or funded by any drug company. and left periaortic lymph nodes, which provided a possible explanation for the backflow of lymph into the pericardium. ${ }^{1}$

Case Report. Patient information. A 33-year-old woman who was previously healthy was referred to our medical institute for evaluation of cardiomegaly that had been detected in a routine, pre-employment chest radiograph (Figure 1). The patient had no cough, sputum, dyspnea, chest pain, or swelling of the lower limbs. She had no fever, no rheumatological conditions, and no past history of tuberculosis, brucellosis, malignancy, or trauma. She was a nonsmoker.

Her clinical physical examination was unremarkable. An initial investigation that included complete blood count, measurement of urea and electrolytes, liver and thyroid function tests, lipid profile, and levels of cardiac enzymes, antinuclear antibodies and complement was normal. Tests for Brucella and viral infections (including hepatitis and HIV tests) were negative. A chest radiograph revealed a boot-shaped cardiomegaly with a clear lungs field. An electrocardiogram showed low voltage waves. Further, an echocardiogram (Figure 2) revealed moderate-to-severe pericardial effusion, with no cardiac tamponade. Chest computed tomography scan confirmed the previous observation of pericardial effusion and showed no evidence of malignancy or tuberculosis. Pericardiocentesis was performed via insertion of a pericardial drain (Figure 3). In total, $850 \mathrm{ml}$ of white milky fluid was drained. Assessment of the drained fluid revealed a white blood cell count of $4,388 \mathrm{cell} / \mathrm{mm}^{3}$, with lymphocyte levels at $97 \%$. Further, gram staining and staining for acidfast bacilli were negative. Cultures for ordinary and tuberculosiscausing bacteria and fungi were negative. A polymerase chain reaction-based test for tuberculosis was negative. Flow cytometry testing for malignancy and lymphoma was also negative. Lymphoscintigraphy lymphangiogram with technetium $99 \mathrm{~m}$ nanocolloids was performed to evaluate the drainage of the lymphatic system (Figure 4). It revealed 1) an abnormal trace accumulation of fluid in the pericardial space, which was consistent with chylopericardium; 2) normal drainage of both lower limbs up to the inguinal lymph nodes and right iliac lymph nodes; and 3) mild decreases in the level of drainage of the left iliac and left periaortic lymph nodes, which could be related to lymphatic obstruction. A diagnosis of idiopathic chylopericardium was made based on the high lymphocytic count and color of drained fluid and lymphoscintigraphy results.

Therapeutic intervention. The patient was prescribed a diet low in fat and high in medium-chain 


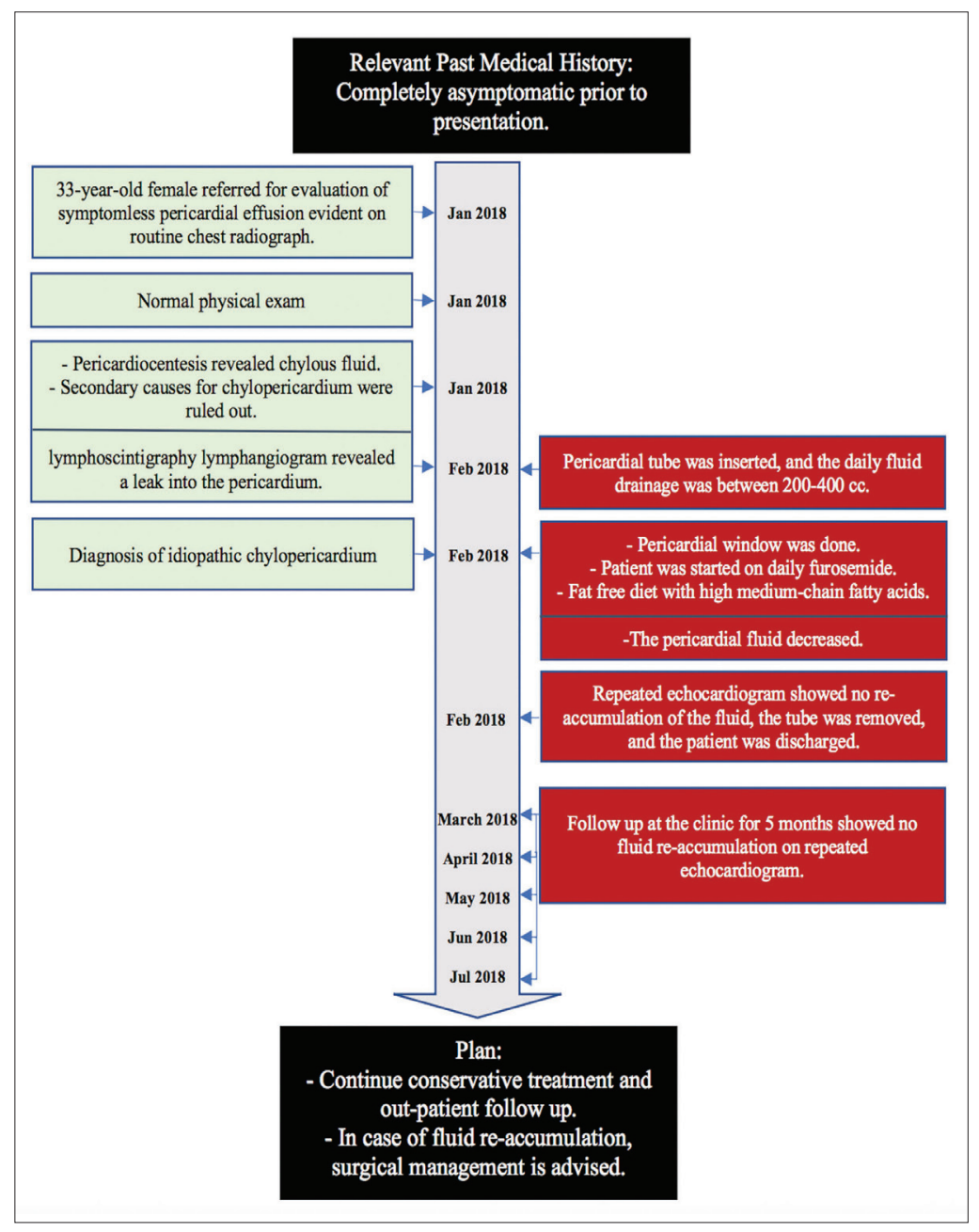

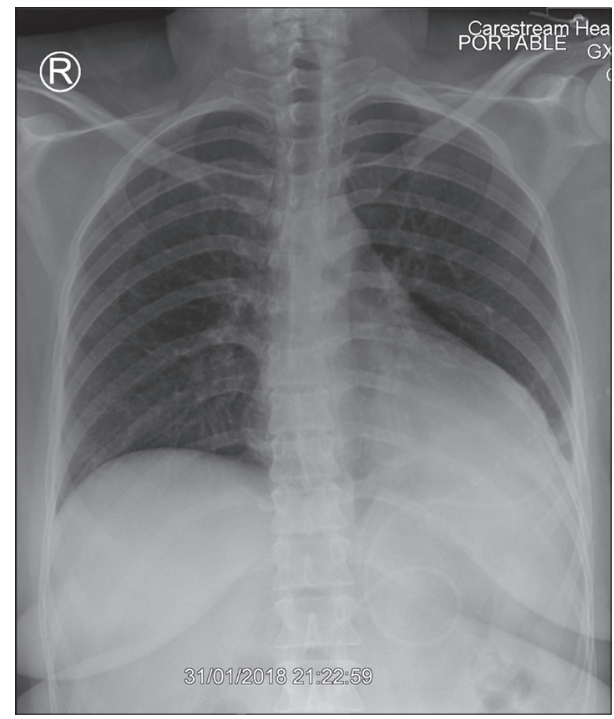

Figure 3 - A chest radiograph revealing an enlarged cardiac silhouette, a pericardial drain is noted in place. The lungs appear unremarkable apart from left basal atelectasis.

Figure 1 - Timeline of the studied participant.
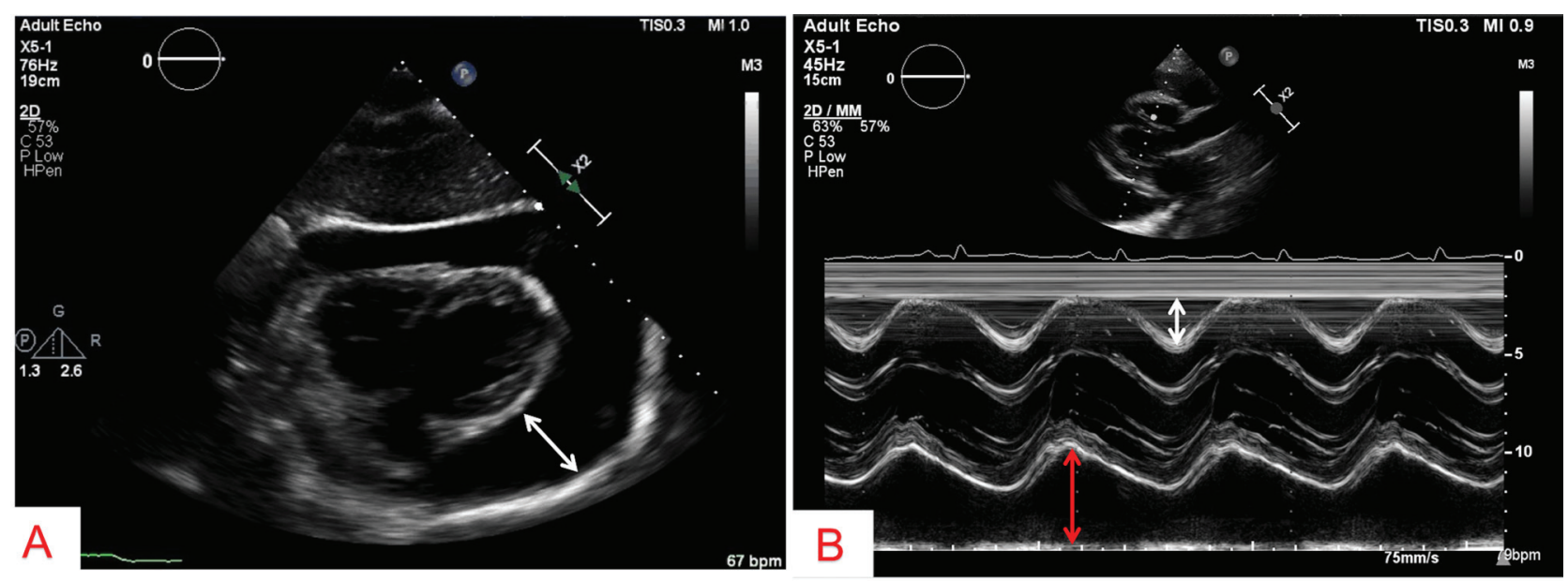

Figure 2 - Echocardiogram of the patient at presentation. A) Subcostal 4 chamber view with white arrow representing large circumferential pericardial effusion. B) M-Mode echocardiogram with white arrow represents early diastolic right ventricular collapse, and the red arrow represents large posterior pericardial effusion. 


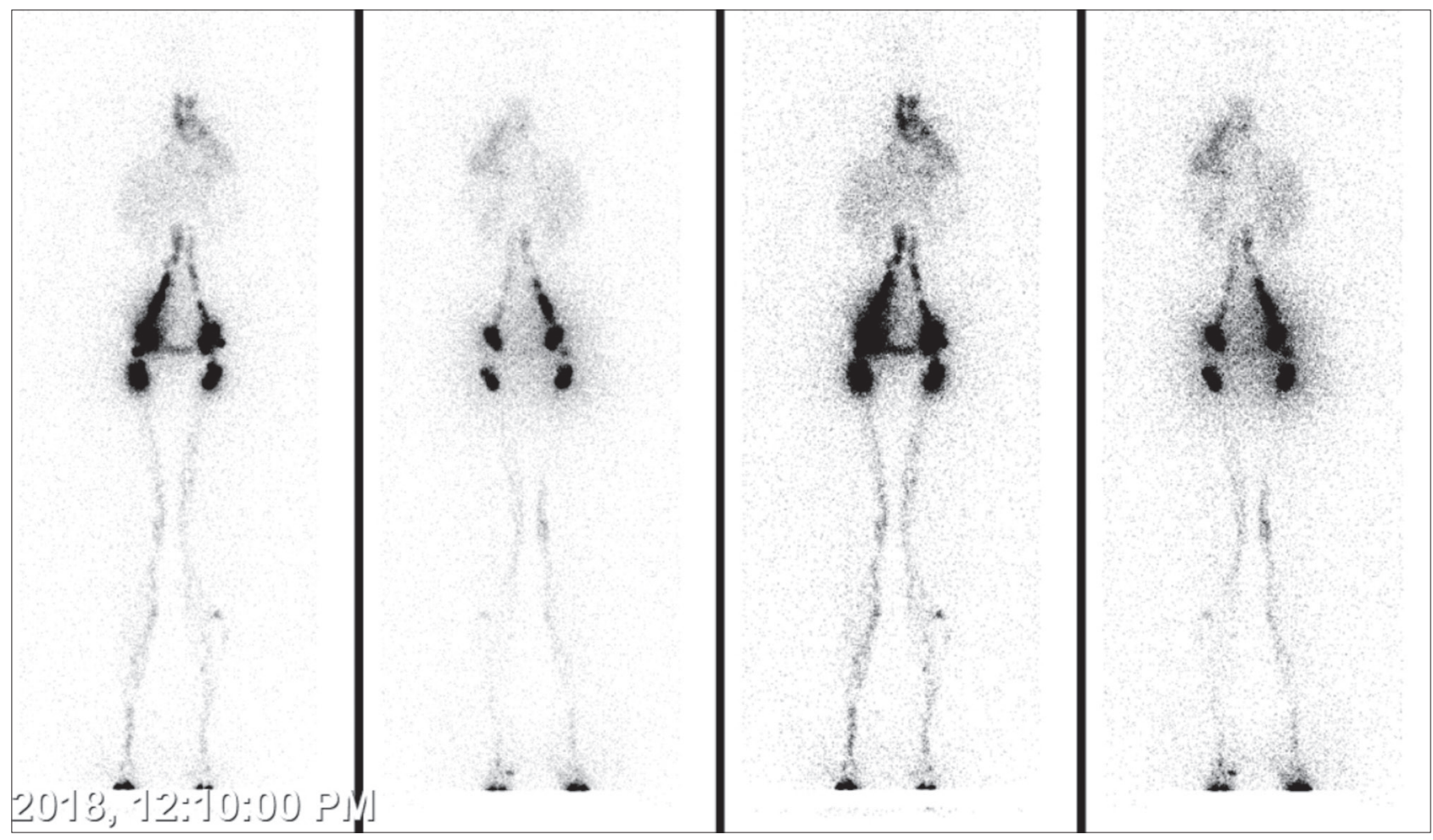

Figure 4 - NM-lymphatic-lymphangiogram scintigram showing abnormal trace accumulation of chyle in the pericardial space, a representative feature of chylopericardium.

triglecerides, and was prescribed a $40 \mathrm{mg}$, oral dose of a furosemide diuretic to be taken daily. The pericardial tube continued to drain 400-500 cc of fluid daily for the following 5 days. Therefore, a pericardial window procedure and biopsy were performed, and a biopsy was sent for histopathological examination which was negative for malignancy and granulomas. After the pericardial window procedure, the daily drainage decreased to $50 \mathrm{cc}$, at which point the pericardial tube was removed with no clinical or radiological evidence of re-accumulation of the fluid. The patient was discharged and returned home with instructions to eat a low-fat diet rich in medium-chain triglycerides, as high fatty meals increase chyle production. ${ }^{1}$

Follow up and outcomes. The management plan for the patient was to continue conservative treatment. In case of the re-accumulation of chylopericardium, the patient would be referred to a specialized center for closure of the thoracic duct. She followed up regularly with the outpatient department for 5 months following the procedure, and a follow-up echocardiogram showed no reaccumulation of chylopericardium. After that point, unfortunately, she did not continue to follow-up.

The case report was conducted ethically in accordance with the World Medical Association Declaration of
Helsinki. Informed consent was not obtained from the patient due to no communication.

Discussion. Idiopathic chylopericardium is a rare clinical condition, the clinical presentation of which is related to the severity of the disease. ${ }^{2}$ Secondary causes of chylopericardium must be excluded before the diagnosis of idiopathic chylopericardium can be made. ${ }^{2}$ Once diagnosed, treatment options must be carefully considered. In patients who are hemodynamically stable, conservative management should be attempted before opting for surgery., ${ }^{3,4}$ Since our patient was hemodynamically stable, conservative management of the disease was implemented; first applying changes to diet, a pericardial drain, and a pericardial window procedure. In case re-accumulation of chylopericardium occurs, the patient would be referred to a specialized center for closure of the thoracic duct. However, the patient responded well with no re-accumulation of pericardial fluid for 5 months following discharge. Chylopericardium occurs when there is a backflow of lymphatic fluid from the thoracic duct to the pericardial space. This is due to high pressure, an abnormal lymphatic vessel wall with valve incomitance, lymphatic obstruction, or lymphangiectasis. ${ }^{1}$ In our patient, 
the lymphoscintigraphy lymphangiogram revealed a decreased level of drainage at the left iliac and left periaortic lymph nodes, which suggested that there may have been lymphatic obstruction.

Patient perspective. Upon admission, the patient was concerned about her cardiomegaly as she was told. She was completely asymptotic. Initial investigations revealed chylopericardium. The condition and the possible causes were explained to the patient and her family. Further investigations excluded secondary causes. After the pericardial drain insertion and the continuous daily drainage of large quantities of chylous fluid, both the patient and her family were more worried. The natural condition of the disease and the treatment strategies were explained to them. Conservative management was the initial step in the form of low fat high medium chain triglycerides and diuretics, then pericardial window biopsy was performed when no response to diet alone. The pericardial fluid drainage decreased and eventually stopped. In case of re-accumulation of the fluid, the patient was informed about referral to a specialized center for surgical closure of the thoracic duct. The patient was discharged happy and was following in the clinic for few months without evidence of fluid re-accumulation.

In conclusion, primary chylopericardium is a rare condition. In countries were tuberculosis is an endemic disease, patients with pericardial effusion are sometimes treated empirically with anti-tuberculosis antibiotics on a trial basis if no other cause for effusion is confirmed. Thus, it is very crucial to consider assessment of the pericardial fluid for chyle to prevent delays in the diagnosis of chylopericardium.

Acknowledgment. We would like to thank the cardiologist Dr. Ahmed Kassem for performing and reporting the echocardiogram, Dr. Nadia Batawil for performing and reporting the lymphoscintigraphy lymphangiogram, and Dr. Lamia Ghazi Jamjoom for performing and reporting the chest radiograph for our patient. We would also like to thank Editage (www.editage.com) for English language editing.

\section{References}

1. Yu X, Jia N, Ye S, Zhou M, Liu D. Primary chylopericardium: A case report and literature review. Exp Ther Med 2018; 15: 419-425.

2. Groves LK, Effler DB. Primary Chylopericardium. $N$ Engl J Med 1954; 250: 520-523.

3. Hsu CY, Huang LY, Chan JS, Yang CC, Hsiao PJ. Chylopericardium. QJM 2018; 111: 203-204.

4. Shah A, Jasani M, Shah A. Pediatric chylopericardium: Treatment conundrum. J Indian Assoc Pediatr Surg 2018; 23: 51-52.

5. O’Donnell J, Kirkham J, Monteith D, Frontario C, Sharma R, Higgins B. Chylous cardiac tamponade with chylothoraces secondary to hodgkin's lymphoma: Octreotide in conjuncture with standard of care dietary fat restriction. Case Rep Crit Care 2019; 2019: 1406840.

6. Willner DA, Kiel J. Pericardial Effusion. [Updated 2019 Dec 17]. In: StatPearls [Internet]. Treasure Island (FL): StatPearls Publishing; 2019. 